\title{
Superior Frontal Sulcus
}

National Cancer Institute

\section{Source}

National Cancer Institute. Superior Frontal Sulcus. NCI Thesaurus. Code C33676.

A groove on the surface of each frontal lobe of the brain that separates the superior frontal gyrus from the middle frontal gyrus. 